\title{
Online Epileptic Seizure Prediction Using Phase Synchronization and Two Time Characteristics: SOP and SPH
}

\author{
Hesam Shokouh Alaei $^{\mathbf{1}^{\circledR}}$, Mohammad Ali Khalilzadeh ${ }^{1}$, Ali Gorji ${ }^{\circledR}$ \\ ${ }^{1}$ Research Center of Biomedical Engineering, Islamic Azad University of Mashhad, Mashhad, 9187147578, Iran \\ ${ }^{2}$ Epilepsy Research Center, University of Munster, Munster, 48149, Germany
}

\begin{abstract}
Background: The successful prediction of epileptic seizures will significantly improve the living conditions of patients with refractory epilepsy. A proper warning impending seizure system should be resulted not only in high accuracy and low false-positive alarms but also in suitable prediction time.

Methods: In this research, the mean phase coherence index used as a reliable indicator for identifying the preictal period of the 14-patient Freiburg EEG dataset. In order to predict the seizures on-line, an adaptive Neuro-fuzzy model named ENFM (evolving neuro-fuzzy model) was used to classify the extracted features. The ENFM trained by a new class labeling method based on the temporal properties of a prediction characterized by two time intervals, seizure prediction horizon (SPH), and seizure occurrence period (SOP), which subsequently applied in the evaluation method. It is evident that an increase in the duration of the SPH can be more useful for the subject in preventing the irreparable consequences of the seizure, and provides adequate time to deal with the seizure. Also, a reduction in duration of the SOP can reduce the patient's stress in the SOP interval. In this study, the optimal SOP and SPH obtained for each patient using Mamdani fuzzy inference system considering sensitivity, false-positive rate (FPR), and the two mentioned points, which generally ignored in most studies.

Results: The results showed that last seizure, as well as 14-hour interictal period of each patient, were predicted on-line without false negative alarms: the average yielding of sensitivity by $100 \%$, the average FPR by 0.13 per hour and the average prediction time by 30 minutes.

Conclusion: Based on the obtained results, such a data-labeling method for ENFM showed promising seizure prediction for online machine learning using epileptic seizure data. Apart from that, the proposed fuzzy system can consider as an evaluation method for comparing the results of studies. Keywords: Online seizure prediction; Mamdani fuzzy inference system; Neuro-fuzzy model; Phase synchronization
\end{abstract}

\author{
*Correspondence to \\ Hesam Shokouh Alaei, \\ Research Center of Biomedical \\ Engineering, Islamic Azad \\ University, Mashhad Branch \\ Post Code: 9187147578; \\ Tel: +98-915-3259380; \\ Email: \\ h.sh.alaei@mshdiau.ac.ir
}

Published online December 13,2019

Citation: Shokouh Alaei H, Khalilzadeh MA, Gorji A. Online epileptic seizure prediction using phase synchronization and two time characteristics: SOP and SPH. Int Clin Neurosci J. 2020;7(1):16-25. doi:10.15171/icnj.2020.03.

\section{Introduction}

Epilepsy is considered one of the most common neurological disorders affecting by $1 \%$ of the world population, which abnormally and spontaneously resulted from excessive electrical discharge in the cerebral cortex. ${ }^{1}$ For epilepsy patients who are not able to control the seizure completely, it strikes suddenly in an unanticipated way that shows one of the most disabling aspects of the illness. Except for the risk of critical injury, there is often a feeling of helplessness that strongly affects the regular daily life of a patient. Hence, if a robust method is capable of predicting the seizure onset accurately, the quality of life for epilepsy patients and therapeutic possibilities can be significantly improved. ${ }^{2}$

Detection of a preictal period considered as an overall basis for predicting epileptic seizures. Significant changes in EEG dynamics are reported to be between a few minutes to several hours before the seizure onset that remains an open question in epilepsy research. ${ }^{3-7}$ With the emergence of high-capacity storage, the epileptic seizure centers were able to store complete data for preoperative monitoring. The purpose of these centers was to test and compare the methods presented on a data set. Most of the seizure prediction results indicate relatively poor performance of univariate measures when they were applied to the long-term database, whereas better results were obtained based on multivariate (bivariate) measures. ${ }^{1,2,8,9}$ The multivariate measurement of time series involves the simultaneous recording of more than one observation over time to evaluate the relationship

(C) 2020 The Author(s). This is an open access article distributed under the terms of the Creative Commons Attribution License (http:// creativecommons.org/licenses/by/4.0/), which permits unrestricted use, distribution, and reproduction in any medium, provided the original work is properly cited. 
between different components of a system. ${ }^{10}$ Based on the hypothesis suggested that seizures generated by unusual synchronization of neurons, many researchers are tried to predict the seizure onset by characterizing the interaction of different brain areas. ${ }^{11}$ They have used bivariate measures in order to predict seizures, such as nonlinear interdependence, phase synchronization, and cross-correlation. ${ }^{12,13}$ Nevertheless, combining the univariate and bivariate nonlinear measures may improve the performance to predict partial seizures in patients with focal neocortical and hippocampal epilepsy. ${ }^{14}$

Lack of a widely accepted methodology to evaluate and compare seizure prediction algorithms considered one of the main seizure prediction challenges. Although the "seizure prediction characteristic" approach has been introduced to evaluate seizure prediction performance, this method is suitable for offline evaluations. ${ }^{15}$ In this approach, a time interval after an alarm, the seizure prediction horizon (SPH) (Figure 1), is required to administer therapeutic intervention or seizure warning devices effectively. Drugs or other treatment strategies can be applied, or the patient behaves cautiously within this time interval. Second, we expect that the seizure should occur after the prediction horizon. However, due to the random features of EEG, seizure onset usually does not occur immediately after the SPH. A new time interval seizure occurrence period (SOP) has been introduced to account for the impossibility of a perfect prediction and to allow a temporal uncertainty in the occurrence of prediction seizures. Accurate prediction defined by the occurrence of a seizure within the SOP ${ }^{5,6}$ Furthermore, the SOP and SPH must also be seriously considered. On the one hand, the SPH should be high enough to provide the patient with adequate time to prepare or prevent the occurrence of the seizure after warning alarm. On the other, the SOP should be low enough to reduce the waiting time in order to decrease the patient's stress. ${ }^{9,16}$

In this paper, the interaction of different brain regions in specific frequency bands has been quantized using longterm intracranial EEG data, through synchronous phase index as a bivariate measure, and then an adaptive Neurofuzzy model was used for classification and prediction. A similar method, "seizure prediction characteristic," has been used to evaluate the output of the model, as in this study, the classifier is trained based on the twotime indices SOP and SPH, which subsequently used in

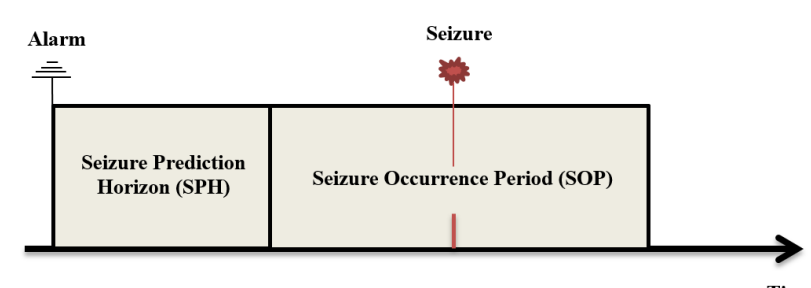

Time

Figure 1. The Illustration of SOP and SPH Intervals. the evaluation approach. Various SOP and SPH values evaluated the output of the model. Optimum SOP and SPH determined by a set of predetermined rules using the Mamdani fuzzy inference system.

\section{Materials and Methods}

Data and Preprocessing

We evaluated our seizure prediction method on the publicly EEG database at the Epilepsy Center of the University Hospital of Freiburg, Germany (http:// epilepsy.uni-freiburg.de/freiburg-seizure-predictionproject/eeg-database) which contains Invasive long-term EEG data, recorded using a Neurofile NT digital-video EEG system with 128 channels, a sampling rate of $256 \mathrm{~Hz}$, and a 16-bit analog-to-digital converter. Their clinical characteristics have summarized in Table 1. In the source dataset, a confirmed neurologist has selected only six channels of EEG dataset in which three electrodes (1-3) chosen over focal areas, and three electrodes (4-6) chosen over nonfocal areas. The EEG recordings for each patient contain separate ictal and interictal datasets, the former including epileptic seizures and at least 50 minutes preictal samples for each seizure, and latter includes almost 24 hours of EEG recording without seizure activity.

In this paper, a sliding window with a $20 \%$ overlap, which consists of 4096 samples (equals 16 seconds), was used to windowing of EEG. Then, in the preprocessing stage, a low-pass filter of the FIR type with a cutoff frequency of $70 \mathrm{~Hz}$ and an order of 18 was used to eliminate the high signal frequencies.

\section{Decomposition of EEG Frequency Bands}

It is necessary to calculate the instantaneous phase of signals in order to get the phase differences of the pair channels of signals. Phase information can be computed by various methods, such as Fourier transform and Wavelet transforms, these methods have a trade-off between the frequency resolution and the temporal resolution. ${ }^{17}$ The Hilbert transform is a well-known method for calculating the instantaneous phase of any signal. It has assumed that the signal contains only one frequency, but this method cannot directly apply to a complex signal, i.e., EEG containing multiple frequencies at any time. ${ }^{18}$ Therefore, the past application of the Hilbert transforms restricted to the single-frequency signal. ${ }^{19} \mathrm{~A}$ new method called the Hilbert-Huang transform (HHT) was introduced to solve this problem based on the combination of empirical mode decomposition (EMD) and Hilbert transform. ${ }^{21}$ Decomposition of EEG to frequency bands based on HHT will compose in 3 steps $^{18,20,21}$ :

1. Decompose the signal into several intrinsic mode functions (IMF) using EMD. In practice, it can show that this decomposition process is complete, adaptive, and local. Figure 2 shows IMFs for singlechannel EEG signals. First, the IMF has the highest frequency contents, and the last IMF has the lowest 
Table 1. Patient characteristics ( $\mathrm{SP}=$ simple partial, $\mathrm{CP}=$ complex partial, $\mathrm{GTC}=$ generalized tonic-clonic, $\mathrm{H}=$ hippocampal, $\mathrm{NC}=$ neocortical)

\begin{tabular}{|c|c|c|c|c|c|c|}
\hline Patient No. & Sex & Age & Seizure type & Seizure origin & \#Seizures & Interictal $(\mathbf{H})$ \\
\hline 1 & $f$ & 15 & $\mathrm{SP}, \mathrm{CP}$ & NC & 5 & 24 \\
\hline 3 & $\mathrm{~m}$ & 14 & $\mathrm{SP}, \mathrm{CP}$ & $\mathrm{NC}$ & 5 & 24 \\
\hline 4 & $f$ & 26 & SP, CP,GTC & $\mathrm{H}$ & 5 & 24 \\
\hline 5 & $f$ & 16 & SP, CP, GTC & $\mathrm{NC}$ & 5 & 24 \\
\hline 9 & $\mathrm{~m}$ & 44 & $\mathrm{CP}, \mathrm{GTC}$ & NC & 5 & 24 \\
\hline 10 & $\mathrm{~m}$ & 47 & SP, CP, GTC & $\mathrm{H}$ & 5 & 24 \\
\hline 11 & $f$ & 10 & SP, CP, GTC & NC & 4 & 24 \\
\hline 12 & $f$ & 42 & SP, CP, GTC & $\mathrm{H}$ & 4 & 25 \\
\hline 14 & $f$ & 41 & $\mathrm{CP}, \mathrm{GTC}$ & $\mathrm{H} \& \mathrm{NC}$ & 4 & 24 \\
\hline 15 & $\mathrm{~m}$ & 31 & SP, CP, GTC & $H \& N C$ & 4 & 24 \\
\hline 16 & $f$ & 50 & SP, CP, GTC & $\mathrm{H}$ & 5 & 24 \\
\hline 18 & $f$ & 25 & $\mathrm{SP}, \mathrm{CP}$ & $\mathrm{NC}$ & 5 & 25 \\
\hline 20 & $\mathrm{~m}$ & 33 & SP, CP, GTC & $\mathrm{NC}$ & 5 & 26 \\
\hline 21 & $\mathrm{~m}$ & 13 & $\mathrm{SP}, \mathrm{CP}$ & $\mathrm{NC}$ & 5 & 24 \\
\hline
\end{tabular}

frequency contents of the signal.

2. Apply the Hilbert transform to each IMF to compute the instantaneous frequency at each sample time. As shown in Figure 2, the EEG signal has 11 IMFs. Hence, there are 11 frequency components in each sample time.

3. For locating delta $(0.5-4 \mathrm{~Hz})$, theta $(4-8 \mathrm{~Hz})$, alpha (8-13), beta (13-30), and gamma (30-70) frequency bands, in each sample, calculates the sum of IMF whose frequency assigned to one of the frequency bands. Figure 3 shows the normalized filtered EEG signal of the one channel and its frequency bands calculated by HHT.

Phase Synchronization Calculation

To describe the interactions of different brain regions, the mean phase coherence has used as a measure of phase synchronization in the proposed method. It characterizes the variations of the phase difference between two separate oscillating systems. ${ }^{9,11}$ The relative phase of such two systems is computed as follows:

$\varphi_{n, m}^{1,2}(\mathrm{t})=\left|\mathrm{n} \phi_{1}(\mathrm{t})-\mathrm{m} \phi_{2}(\mathrm{t})\right|$

Where $\mathrm{m}$ and $\mathrm{n}$ are integers and state that the frequency lock occurs at specific phases of the two oscillating systems, $\phi_{1}(\mathrm{t})$ and $\phi_{2}(\mathrm{t})$ denoting the instantaneous phase of the oscillators computed by the Hilbert transform..$^{22}$ Then, Mean phase coherence of two recording channels 1 and 2 based on the relative phase with a ratio of $n: m=1$ : 1 and a sampling rate of $\frac{1}{\Delta t}$, is defined as:

$\mathrm{R}_{1,2}=\left|\frac{1}{\mathrm{~N}} \sum_{\mathrm{j}=0}^{\mathrm{N}-1} e^{-j \varphi_{1,1}^{1,2}(j \Delta t)}\right|$

For every possible combination of different EEG recording channels $i$ and $j$, the mean phase coherence value

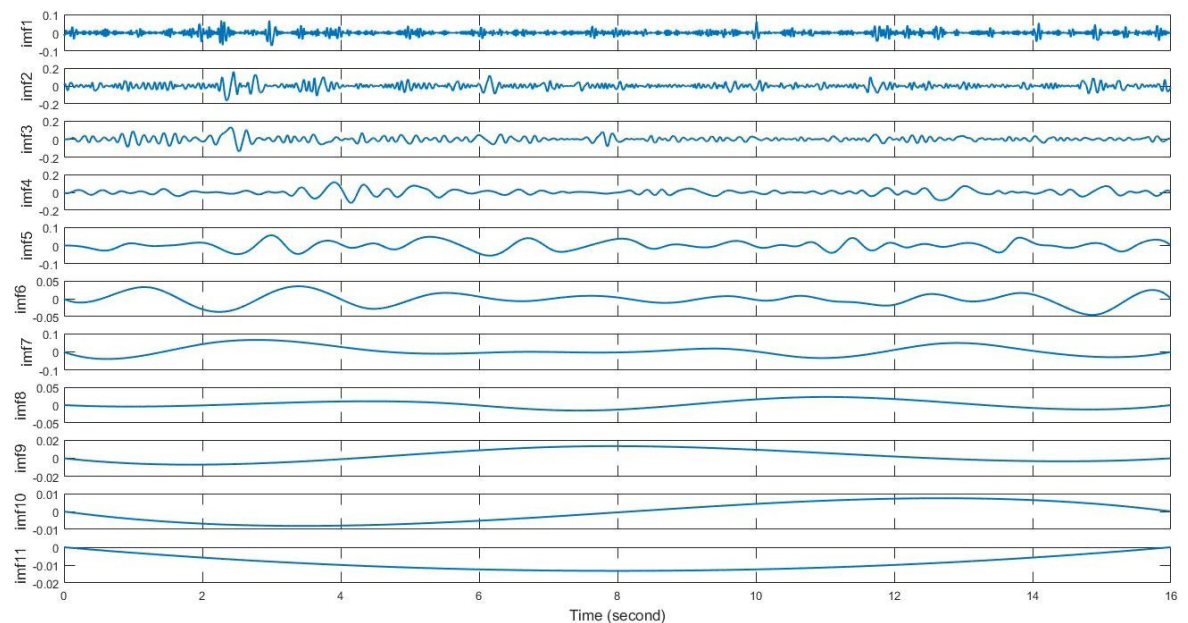

Figure 2. Decomposition of the 16-second (equal to window time) normalized filtered EEG of fifth channel recorded from patient number 1 into 11 components (IMFs) using EMD algorithm. 


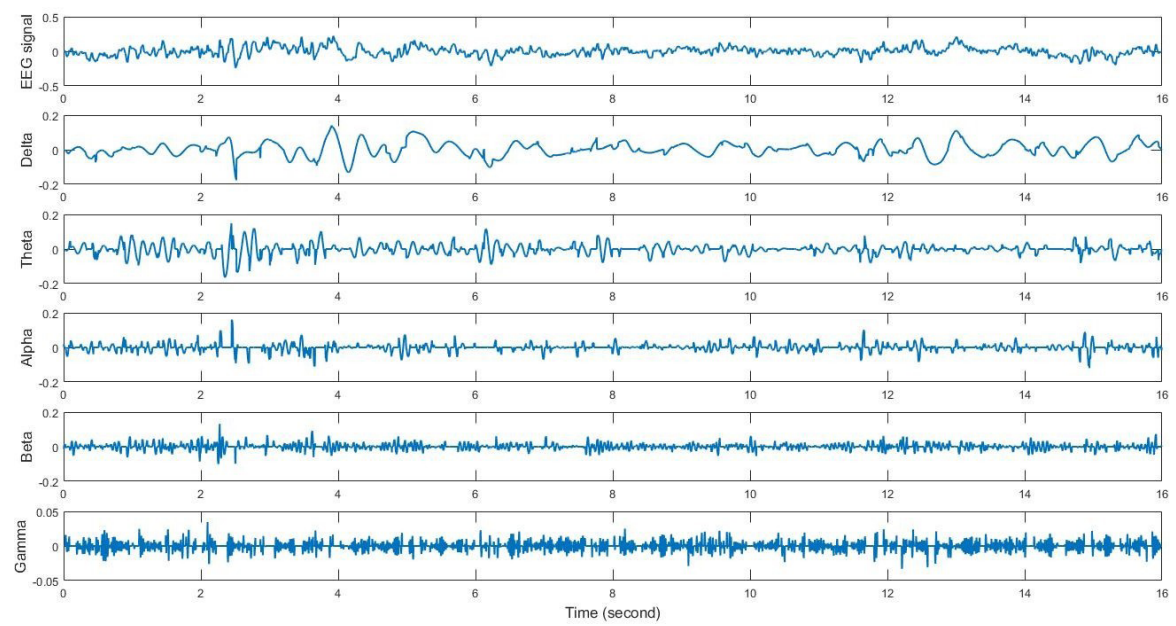

Figure 3. The 16-second normalized filtered EEG signal of fifth channel recorded from patient number 1 and its frequency bands, delta, theta, alpha, beta and gamma, calculated by HHT method. It is clear that slow changes and low frequency of the main signal can observe in the delta band.

related to every pair of frequency bands $f$ was computed for each consecutive window. As in this research, there are 6 channels and five frequency bands, so 75 different values $R_{i, j}^{f}$ were extracted from each window.

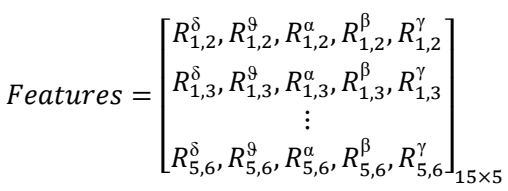

Selecting the Optimal Features

Researchers claimed that considerable dynamic changes in the interaction between brain areas always appeared in some specific brain regions before the occurrence of the seizure. ${ }^{9}$ It indicates that among the 75 values in (3), they should properly select in order to predict epileptic seizures accurately; otherwise, the calculations become more complex, and the possibility of the false alarms increases significantly. So, the number of features should be as low as possible to enhance the performance of seizure prediction. To this end, the magnitude of the relationship between ictal and interictal samples measured by Spearman correlation coefficient test, then the indices that had the least correlation (the correlation coefficient is almost zero) selected as optimal features. ${ }^{23}$

\section{Classification and Online Prediction of Epileptic} Seizures

Most seizure prediction methods are offline approaches, but as for long-term data using offline approaches lead to higher computational cost. In this paper, we take advantage of evolving neuro-fuzzy model (ENFM) for seizure prediction based on recursive fuzzy clustering developing by Soleimani et al. ${ }^{24}$ The Gath-Geva (GG) clustering as the basis for the recursive fuzzy clustering has been utilized in ENFM, since GG algorithm has some advantages over other fuzzy clusterings (e.g., k-means algorithm, Gustafson Kessel clustering) in terms of creating clusters in different shapes and sizes. ${ }^{25,26}$ The ENFM structure is analogous to Takagi-Sugeno fuzzy models, and it is capable of adapting by changes in system behavior by adding new neurons (fuzzy rules) or merging similar existing neurons. Contrary to simple GG algorithm, cluster parameters (e.g., cluster center and covariance matrices) of ENFM are updated using adaptive equations. For more details, refer to Soleimani et al. ${ }^{24}$

The basis of the prediction is that the interval from the time before seizure onset continuously alerted to the patient. The length of this interval is equal to the prediction time $(P)$. The block diagram in Figure 4 shows the algorithm. Every consecutive window is labeled based

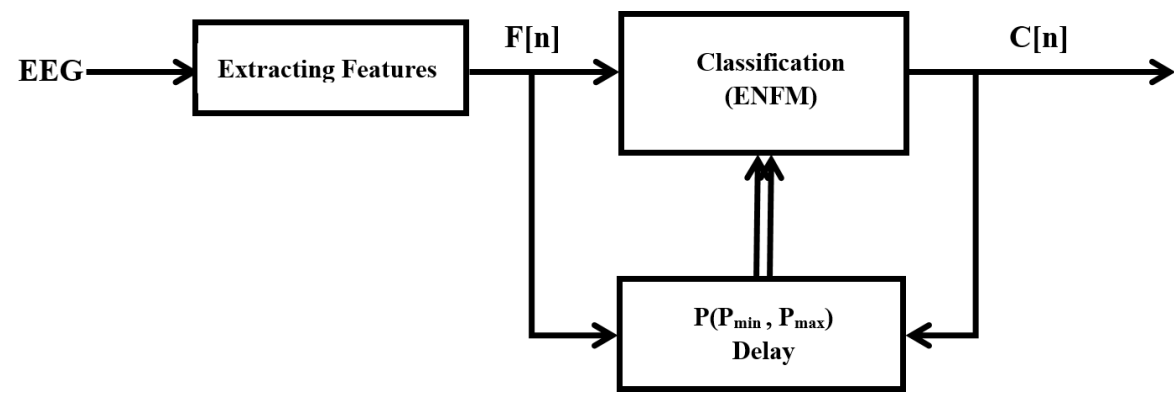

Figure 4. After feature extraction, the ENFM predicts seizure occurrence period (SOP) $p_{\min }(\mathrm{SPH})$ minutes ahead. The model trained at the nth window based on feature $F\left[n-p_{\max }\right]$ and class label $C\left[n-p_{\max }\right]$. 
on the occurrence of a seizure $p_{\max }$ minutes later ( 1 for "seizure," and 0 for "no seizure"), yielding a sequence $F[n]$ of features related to the $\mathrm{n}$ th temporal window with corresponding labels $C[n]$. Since in online training ENFM, the duration of preictal and interictal is unknown, every such 16 s windows are labeled $0(C[n]=0)$, representing of the interictal period, unless the seizure to have occurred in a specific window. In this case, concerning the twotime characteristics specified in the evaluation method of this paper, windows for as long as $p_{\max }-p_{\text {min }}$ (SOP) to $p_{\text {min }}$ $(\mathrm{SPH})$ before seizure onset are labeled $+1(C[n]=1)$ (see Figure 5). Thus, there is a $p_{\max }(\mathrm{SOP}+\mathrm{SPH})$ delay between the training data and the test input sample, so that the ENFM trained at the $\mathrm{n}$ th window based on the input $\mathrm{F}$ $\left[n-p_{\max }\right]$ and the output C $\left[n-p_{\max }\right]$.

Adaptation of model to evaluation method in order to increase seizure prediction accuracy considered as a reason for defining this class labeling approach. As shown in Figure 5, for every alarm that rises in the color window $\left(p_{\max }-p_{\min }\right)$, the seizure onset will occur within the SOP span following SPH interval, while alarms that take place outside the color window are all considered as the false positive prediction. Therefore, the model is expected to train for each SOP and SPH properly.

\section{Post-processing}

The number of false predictions should be controlled, of which too many cannot be accepted, e.g., for a seizure warning device due to the loss of patients' confidence. Here a simple algorithm is proposed to minimize false prediction. Although raw ENFM output is between 0 and +1 , it is possible to set an absolute value of 0 or +1 for each output sample. We achieved this requirement by moving average filter with a length window of 10 minutes. In this algorithm, the proposed model detects the SOP after the SPH for every 10 minutes. If the mean value of each window to consecutively increased four-times, then the alarm would rise, and it will stay on until the mean value to consecutively decreased two-times afterward. This algorithm, which is useful in online processes, makes output sensitive to the slope of amplitudes changes instead of amplitudes itself.

\section{Model Evaluation}

The performance of seizure prediction is possible by statistical criteria such as sensitivity and specificity, whereas specificity has defined in the form of falsepositive rate (FPR) (4), (5):

Sensitivity $=\frac{\mathrm{TP}}{\mathrm{TP}+\mathrm{FN}}$

$\mathrm{FPR}=\frac{\mathrm{FP}}{\mathrm{TN}+\mathrm{FP}}$

Given the predetermined SOP and SPH intervals, we can divide the output model into one of the following four

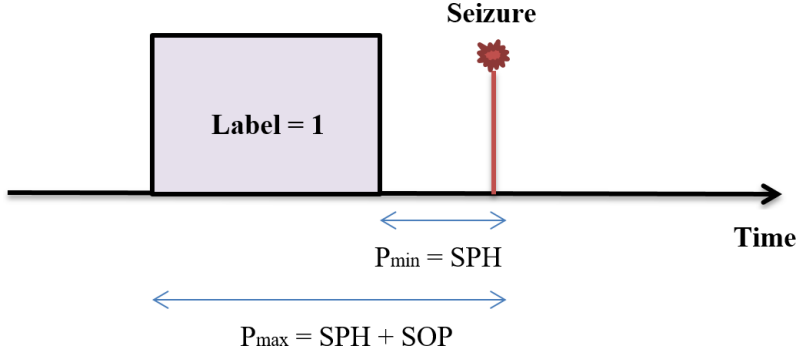

Figure 5. Labeling the ENFM output in the online process when the seizure starts.

categories:

1. If $C[n]=1$ and at least one seizure occur within the SOP duration after the SPH interval, then it is the true positive result.

2. If $C[n]=1$ and any seizure do not occur within the SOP duration after the SPH interval, then it is a falsepositive result.

3. If $C[n]=0$ and any seizure do not occur within the SOP duration after the SPH interval, then it is a true negative result.

4. If $C[n]=1$ and at least one seizure occur within the SOP duration after the SPH interval, then it is a false negative result.

\section{Optimal SOP and SPH Selection}

As learning of the ENFM based on SOP and SPH length, these two intervals should be properly selected. Two terms are critical in determining these values. First, a proper assessment of seizure prediction performance is vital for seizure prediction system in clinics. A good clinical application requires an algorithm with high sensitivity but low FPR. ${ }^{27}$ Second, considering that an increase in SPH can be more beneficial for the patients if they allow themselves to retreat from public or potentially dangerous situations or even to take action against seizures while decreasing SOP would reduce the patients' stress, and it leads to minimizing physiological disadvantages caused by potential side effects of drugs or long term electric stimulation of focal brain structures.

Mamdani fuzzy inference system, which is a nonlinear mapping that derives its output based on fuzzy reasoning, was applied to achieve unique SOP and SPH values among different intervals. ${ }^{28}$ Firstly, according to two terms that have mentioned in this paper, predefined rules are specified as follow:

1. If SOP is low and SPH is high, and Sensitivity is high, and FPR is low, then the result will be Excellent.

2. If SOP is low and SPH is low, and Sensitivity is high, and FPR is low, then the result will be Good.

3. If SOP is high and SPH is high, and Sensitivity is high, and FPR is low, then the result will be Good.

4. If SOP is high and SPH is low, and Sensitivity is high, and FPR is low, then the result will be Acceptable. 
5. If Sensitivity is low or FPR is high, then the result will be Poor.

According to Figure 6, Gaussian function has been used as membership function of four input variables $x=\left\{x_{1}, x_{2}\right.$, $\left.x_{3}, x_{4}\right\}$, Sensitivity $\left(x_{1}\right)$, FPR $\left(x_{2}\right)$, SOP $\left(x_{3}\right)$ and SPH $\left(x_{4}\right)$. Each of the input variables consists of 2 Gaussian fuzzy sets, low $(L)$ and high $(H)$ corresponding membership functions $\mu_{L}(x)$ and $\mu_{H}(x)$ as defined:

$$
\begin{aligned}
& \mu_{L_{j}}\left(x_{j}\right)=\exp \left[-\left(\frac{\left(x_{j}-c_{j}^{L}\right)}{\delta_{j}^{L}}\right)^{2}\right] \text { for } j=1,2,3,4 \\
& \mu_{H_{j}}\left(x_{j}\right)=\exp \left[-\left(\frac{\left(x_{j}-c_{j}^{H}\right)}{\delta_{j}^{H}}\right)^{2}\right] \text { for } j=1,2,3,4
\end{aligned}
$$

Where $c_{1}^{L}=0 ; \delta_{1}^{L}=30 ; c_{1}^{H}=100 ; \delta_{1}^{H}=25 ; c_{2}^{L}=0 ; \delta_{2}^{L}$ $=0.13 ; c_{2}^{H}=1 ; \delta_{2}^{H} 0.3 ; c_{3,4}^{L}=5 ; \delta_{3,4}^{L}=7 ; c_{3,4}^{H}=30 ; \delta_{3,4}^{H}=7$;

According to Figure 7, four triangular functions have used as the output variable membership function (Result). Since the output of the fuzzy system belongs only to one of the four categories (Excellent, Good, Acceptable, Poor), no overlap considered between membership functions. The output range of the result variable defined from 0 to 40. Thus, the Poor, Acceptable, Good, Excellent groups ranged from 0 up to 10,10 up to 20,20 up to 30 , and 30 up to 40 , respectively.

The fuzzy inputs applied to the antecedents of the fuzzy rules. Firing strength of each rule has been calculated through the min operator as a t-norm operation for evaluating conjunction of the 1-4 rules, similarly, the max operator as a t-conorm operation for evaluating the disjunction of the fifth rule. The implementation of the inference rules is done using the compositional rule of inference. Then, the final output is calculated by defuzzification using Centre of Gravity (CoG) method by aggregation of the five individual output fuzzy sets. ${ }^{29}$

In this study, considering the patient needs an early epileptic seizure of 3 to 5 minutes, we set the minimum SPH interval of 5 minutes. ${ }^{6,30,31}$ When the seizure prediction method works well, a much smaller SOP may be eligible (for example, for a seizure warning device). ${ }^{6}$ Therefore, the minimum SOP period set to 5 minutes. However, an SOP for half an hour is also reasonable if the treatment effect lasts for this period, in which maximum SOP set to 30 minutes. For example, this has usually expected for antiepileptic drugs. ${ }^{6}$ Unlike Aarabi et al, Baghdadi et al, Arthurs et al and Zhang et al, SPH interval as extended as found in this study would definitely leave enough
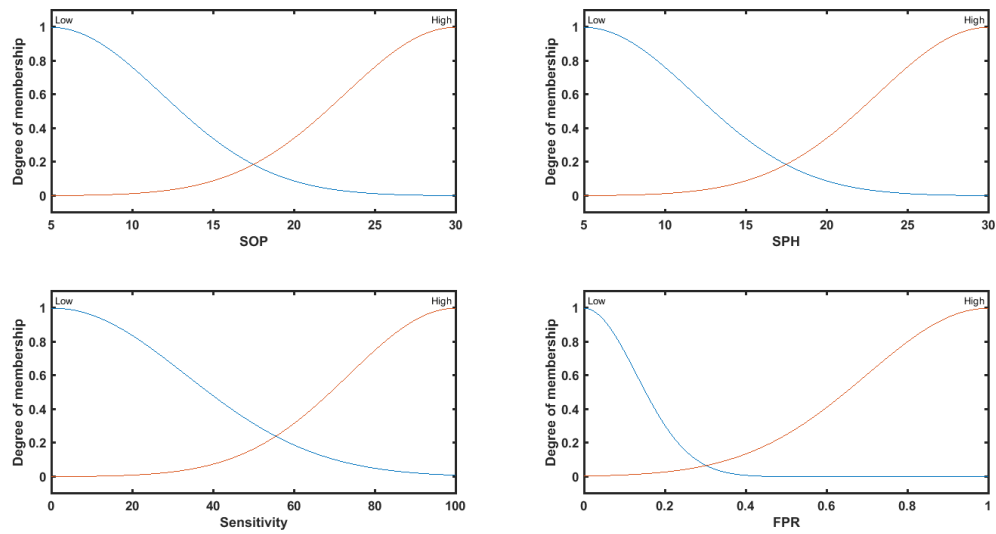

Figure 6. The designed membership functions of the four input variables of the fuzzy inference system

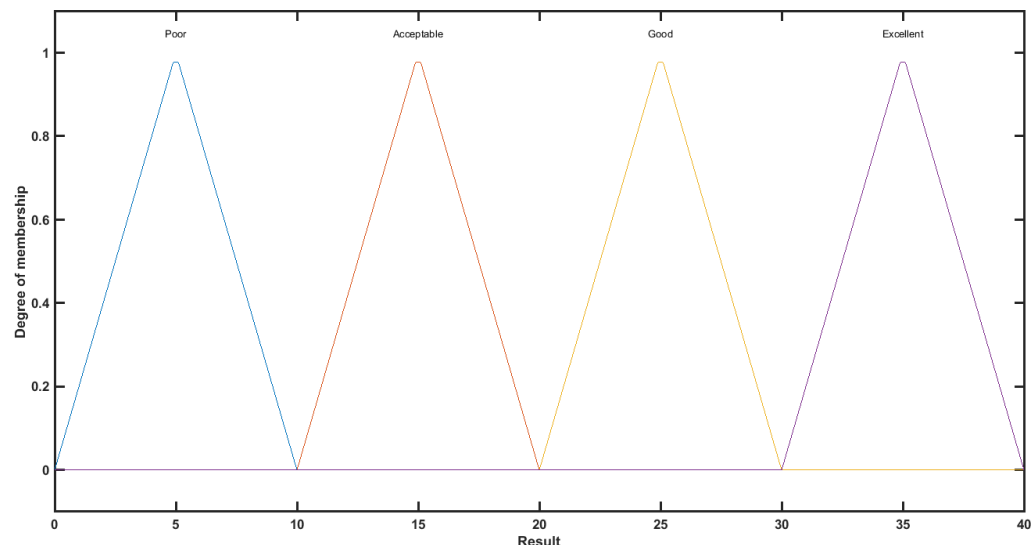

Figure 7. The designed membership functions of the output variable of the fuzzy inference system. 
time for possible strategies to prevent the irreparable consequences of seizure, for example, a patient is driving on a highway or swimming in the pool, consequently, requires more time to leave such situations. ${ }^{14,21,30,32}$ Thus, we set maximum SPH to 30 minutes, which seems an adequate time for the patient to behave more efficiently.

\section{Results}

The small number of seizures for every patient brings about an inappropriate on-line training ENFM as the dataset contains at most five seizures for each patient, so only patients having 4 or 5 seizures were considered for evaluation (14 patients selected for evaluation in total).

In order to test the concept of on-line training, the ictal dataset set to a long-term interictal dataset (i.e., the duration of preictal is at least 50 minutes per seizure). Since in online training, the ENFM trained by past samples and tested by the present sample, it is impossible to divide the entire data, such as offline training, into in-sample data and out-of-sample data, so by overtime, the ENFM results in high sensitivity but low FPR. For having an accurate evaluation, half of the total dataset for each patient (10hour interictal samples with preictal samples of the first 3 or 4 seizures) is considered only for on-line training, but remaining dataset ( 14 hours interictal samples with preictal samples of the last seizure) is accounted for evaluation as it undergoes on-line training.

As shown in Figure 8, the model trained with $\mathrm{SOP}=5$ and $\mathrm{SPH}=20$ minutes. The last seizure predicted without a false negative alarm, along with the good FPR. The first three positive alarms are complete nonsense in interictal duration and defined as a pure false positive alarm. In this particular subject, the preictal period is equal to 95 minutes before the seizure onset. As can be seen, the fourth positive alarm is shown about half an hour before the seizure. Even though there was no such seizure within 5 minutes for the next 20 minutes, this alarm considered a false-positive prediction, although the next two positive alarms considered as a true positive prediction, the rest of the positive alarms due to a warning after the seizure defined as false positive predictions.

In order to achieve best SOP and SPH for each patient, the values of sensitivity and FPR calculated for different SOPs, and SPHs ranged from 5 up to 30 minutes with step 5 minutes, yet the results of two patients obtained with SOP and SPH ranged from 10 up to 60 minutes with step by 10 minutes. Then, the values of sensitivity and FPR in addition to their corresponded SOP and SPH gave as inputs to the fuzzy inference system. According to fuzzy group results, whose scores are between 0 up to 40, the SOP and the SPH with the highest score selected as optimal intervals.

The optimum SOP and optimum SPH for each patient, along with sensitivity and FPR, have been summarized in Table 2. On average, the SOP by 7 minutes and the SPH by 27 minutes obtained for the entire group of patients, with sensitivity by $100 \%$ and FPR by 0.13 per hour. The Excellent fuzzy system output for most patients indicates that the fuzzy system inputs adapted to desired conditions, such as low SOP, high SPH, high sensitivity, and low FPR. However, the fuzzy system output of four patients $(5,9$, $16,20)$ is lower than the Excellent result. The reason is that the SPH interval does not match with our expected indicator, while other parameters are following the terms.

The prediction time that defined as an interval between the first true positive alarm and seizure onset has also reported for every patient in Table 2 . As we expected, all prediction times varied between $\mathrm{P}_{\min }$ and $\mathrm{P}_{\max }$ periods. Another interesting point is that all optimal SPHs are relatively equal to prediction times. As on average, the best SPH had almost the same value of prediction time with 27 minutes and 30 minutes, respectively.

\section{Discussion and Conclusion}

In this study, a new patient-specific model has presented for the prediction of epileptic seizures from the online analysis of EEG. The interaction of different brain regions obtained through the phase synchronization of the EEG

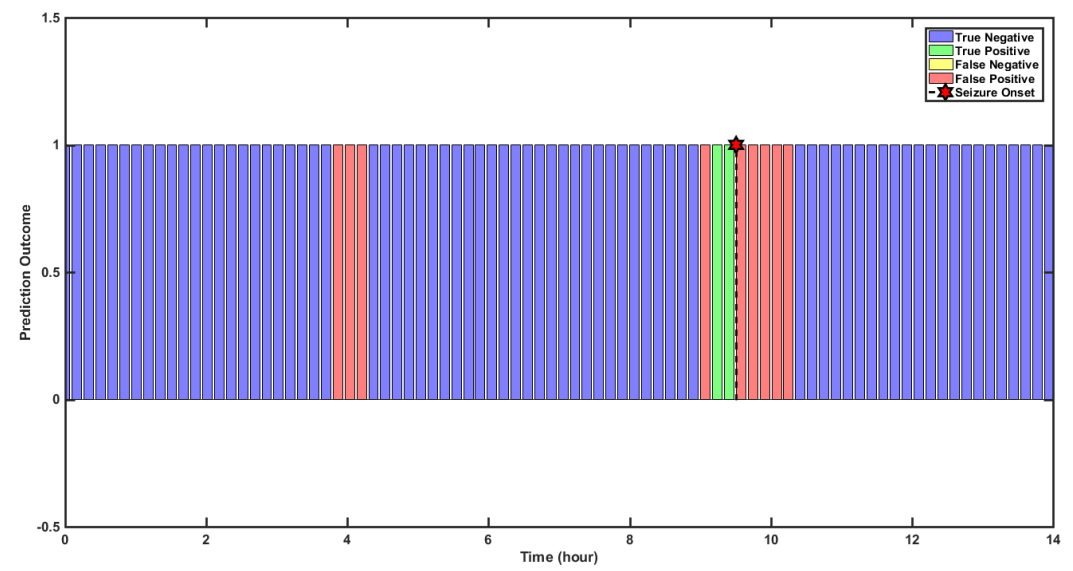

Figure 8. The outcome of the proposed model for patient number 18 ( $\mathrm{SOP}=5, \mathrm{SPH}=20$, Sensitivity=100\%, FPR=0.11 per hour). Each output sample represents 10 minutes 
Table 2. Prediction results of 14 patients using the proposed algorithm

\begin{tabular}{|c|c|c|c|c|c|c|}
\hline Patient No. & Best (min) & SOP, Best (min) & SPH, Sensitivity & FPR & Prediction Time (min) & Fuzzy (Score) \\
\hline 1 & 10 & 30 & $100 \%$ & 0.12 & 39 & Excellent (33.83) \\
\hline 3 & 5 & 30 & $100 \%$ & 0.08 & 30 & Excellent (34.38) \\
\hline 4 & 10 & 30 & $100 \%$ & 0.16 & 38 & Excellent (33.15) \\
\hline 5 & 5 & 10 & $100 \%$ & 0.1 & 13 & Good (24.8) \\
\hline 9 & 5 & 5 & $100 \%$ & 0.22 & 8 & Good (22.33) \\
\hline 10 & 5 & 25 & $100 \%$ & 0.12 & 29 & Excellent (33.83) \\
\hline 11 & 10 & 60 & $100 \%$ & 0.12 & 66 & Excellent (34.06) \\
\hline 12 & 5 & 25 & $100 \%$ & 0.19 & 26 & Excellent (32.19) \\
\hline 14 & 5 & 20 & $100 \%$ & 0.09 & 22 & Excellent (31.93) \\
\hline 15 & 10 & 60 & $100 \%$ & 0.15 & 65 & Excellent (33.6) \\
\hline 16 & 5 & 15 & $100 \%$ & 0.15 & 18 & Good (26.26) \\
\hline 18 & 5 & 20 & $100 \%$ & 0.11 & 21 & Excellent (31.78) \\
\hline 20 & 5 & 15 & $100 \%$ & 0.16 & 17 & Good (26.17) \\
\hline 21 & 10 & 30 & $100 \%$ & 0.09 & 32 & Excellent (34.10) \\
\hline Average & $\approx 7$ & $\approx 27$ & $100 \%$ & $\approx 0.13$ & $\approx 30$ & \\
\hline
\end{tabular}

channels pairs in the specific frequency domains. We used the mean phase coherence indicator to distinguish between preictal and interictal periods. Best features were extracted by Spearman correlation coefficient test to minimize the complexity of the calculation. A recursive extension of the GG clustering algorithm as a basis for online tuning and development of the Neuro-fuzzy model was adaptively applied to predict epileptic seizures. It is noticeable that in the proposed approach, few seizures for each patient make it difficult to assess the seizure prediction i.e., a dataset containing up to 5 seizures per patient. Soleimani et al suggested that in order to compensate for the unbalanced classes, the method of surrogate data is used to generate new preictal samples by perturbing the available data points. ${ }^{25}$ The surrogate data method leads to perturbing the phase of the Fourier transform of the real data points. As a result, the preictal period cannot properly identify by the mean phase coherence indicator. Here we overcome this problem by defining two intervals, SOP and $\mathrm{SPH}$, that were used to train the Neuro-fuzzy model without adding the number of artificial samples generated to the actual data.

We also proposed a novel evaluation method to select optimal SOP and SPH intervals based on predetermined rules that were wisely adjusted based on the patient's safety. The results showed that for the mean SOP by 7 minutes and mean SPH by 27 minutes, the sensitivity and the FPR were equal to $100 \%$ and 0.13 per hour, respectively. This optimization algorithm can also be used to compare other research results. Such a comparison can be made through the rules of the Mamdani fuzzy inference system of this paper, considering the selected SOP, SPH, sensitivity, and false prediction rate of other studies.
In Table 3, the performance of the proposed approach compared with some of the recent achievements in seizure prediction that evaluated with the seizure prediction characteristic method. The values of SOP, SPH, sensitivity, and FPR reported in studies have been given as inputs to the fuzzy inference system. Generally, the range of input variables, SOP and SPH, is considered to be five up to 30 minutes, whereas we observe that some values of SOP and $\mathrm{SPH}$ are out of this range. Thus, we extended the range of input variables from 0 up to 60 minutes without changing the pattern of membership functions for testing those values in which SOP and SPH are outside the range. The score of the proposed approach was superior to others due to the defined rules of the Mamdani fuzzy inference system. The reason for this superiority is because of the major differences in the choice of SOP and SPH intervals. For example, sensitivity by $92.90 \%$ and FPR by 0.09 per hour are the perfect result for Aarabi et al. ${ }^{14}$ However, the evaluation of this method does not justify by 10 seconds of SPH and 50 minutes of SOP. Since in the event of a seizure warning, the patient is at an insufficient time to prepare for seizure, and the patient's anxiety is more than usual when the SOP is too large. According to the results of Hang et al, the length of selected SOP and SPH is far better than the selected SOP and SPH of this study, whereas the sensitivity and the FPR are lower and higher than the sensitivity and FPR of this study, respectively. Consequently, the lower score attributed to the method of Hang et al. ${ }^{33}$

In the context of present neuroscience knowledge, the length of the preictal period is unclear and varies from seizure to next seizure. ${ }^{3}$ This variation may affect the results when a constant SOP and SPH considered for the 
Table 3. Comparison of our approach with other approaches evaluated on Freiburg EEG database

\begin{tabular}{|c|c|c|c|c|c|c|c|}
\hline Authors & Year & Measure & SOP & SPH & Sensitivity & FPR & Fuzzy Group (score) \\
\hline Winterhalderet & 2006 & Phase synchronization & $30 \mathrm{~min}$ & $10 \mathrm{~min}$ & $60 \%$ & 0.15 & Acceptable (11.5) \\
\hline Shao-Hang Hung & 2010 & Wavelet - correlation dimension & $18 \mathrm{~s}$ & $60 \mathrm{~min}$ & $86.96 \%$ & 0.25 & Good (28.13) \\
\hline Shufang Li & 2013 & Spike rate & $50 \mathrm{~min}$ & $10 \mathrm{~s}$ & $72.70 \%$ & 0.1 & Acceptable (13.6) \\
\hline Yang Zheng & 2013 & Phase synchronization & $30 \mathrm{~min}$ & $10 \mathrm{~min}$ & $85 \%$ & 0.15 & Acceptable (14.5) \\
\hline Yanli Zheng & 2014 & Higuchi fractal dimension & $30 \mathrm{~min}$ & $2 \mathrm{~min}$ & $86.95 \%$ & 0.2 & Acceptable(14) \\
\hline Ardalan Aarabi & 2017 & Nonlinear features & $50 \mathrm{~min}$ & $10 \mathrm{~s}$ & $92.90 \%$ & 0.09 & Acceptable (14.9) \\
\hline Our approach & 2019 & Phase synchronization & $7 \mathrm{~min}$ & $27 \mathrm{~min}$ & $100 \%$ & 0.13 & Excellent (33.76) \\
\hline
\end{tabular}

whole EEG. In summary, future work will be devoted to designing an adaptive SOP and SPH optimization algorithm to improve the performance of this epileptic seizure prediction model. Overall, this approach can use for home care applications for which a seizure warning system can be designed that predicts a safe time zone and warning time zone with high accuracy.

\section{Conflict of Interest Disclosures}

The authors declare that they have no conflict of interests.

\section{Ethical Statement}

This article does not contain any studies performed on human or animal participants by any of the authors.

\section{Acknowledgments}

The authors would like to thank the Epilepsy Center of the University Hospital of Freiburg for sharing the EEG database.

\section{References}

1. Litt B, Lehnertz K. Seizure prediction and the preseizure period. Curr Opin Neurol. 2002;15(2):173-7. doi: 10.1097/00019052-200204000-00008.

2. Mormann F, Andrzejak RG, Elger CE, Lehnertz K. Seizure prediction: the long and winding road. Brain. 2007;130(Pt 2):314-33. doi: 10.1093/brain/awl241.

3. Bandarabadi M, Rasekhi J, Teixeira CA, Karami MR, Dourado A. On the proper selection of preictal period for seizure prediction. Epilepsy Behav. 2015;46:158-66. doi: 10.1016/j. yebeh.2015.03.010.

4. Chiang CY, Chang NF, Chen TC, Chen HH, Chen LG. Seizure prediction based on classification of EEG synchronization patterns with on-line retraining and post-processing scheme. Conf Proc IEEE Eng Med Biol Soc. 2011;2011:7564-9. doi: 10.1109/iembs.2011.6091865.

5. Schelter B, Winterhalder M, Maiwald T, Brandt A, Schad A, Schulze-Bonhage A, et al. Testing statistical significance of multivariate time series analysis techniques for epileptic seizure prediction. Chaos. 2006;16(1):013108. doi: 10.1063/1.2137623.

6. Schelter B, Winterhalder M, Maiwald T, Brandt A, Schad A Timmer J, et al. Do false predictions of seizures depend on the state of vigilance? A report from two seizure-prediction methods and proposed remedies. Epilepsia. 2006;47(12):2058-70. doi: 10.1111/j.1528-1167.2006.00848.x.

7. Wang S, Chaovalitwongse WA, Wong S. Online seizure prediction using an adaptive learning approach. IEEE Trans Knowl Data Eng. 2013;25(12):2854-66. doi: 10.1109/ TKDE.2013.151.

8. McSharry PE, Smith LA, Tarassenko L. Prediction of epileptic seizures: are nonlinear methods relevant? Nat Med. 2003;9(3):241-2; author reply 2. doi: 10.1038/nm0303-241.

9. Zheng Y, Wang G, Li K, Bao G, Wang J. Epileptic seizure prediction using phase synchronization based on bivariate empirical mode decomposition. Clin Neurophysiol. 2014;125(6):1104-11. doi: 10.1016/j.clinph.2013.09.047.

10. Carney PR, Myers S, Geyer JD. Seizure prediction: methods. Epilepsy Behav. 2011;22 Suppl 1:S94-101. doi: 10.1016/j. yebeh.2011.09.001.

11. Mormann F, Lehnertz K, David P, E. Elger C. Mean phase coherence as a measure for phase synchronization and its application to the EEG of epilepsy patients. Physica D. 2000;144(3-4):358-69. doi: 10.1016/S0167-2789(00)000877.

12. Arnhold J, Grassberger P, Lehnertz K, Elger CE. A robust method for detecting interdependences: application to intracranially recorded EEG. Physica D. 1999;134(4):419-30. doi: 10.1016/S0167-2789(99)00140-2.

13. Mormann F, Kreuz T, Andrzejak RG, David P, Lehnertz K, Elger CE. Epileptic seizures are preceded by a decrease in synchronization. Epilepsy Res. 2003;53(3):173-85. doi: 10.1016/s0920-1211(03)00002-0.

14. Aarabi A, He B. Seizure prediction in patients with focal hippocampal epilepsy. Clin Neurophysiol. 2017;128(7):1299307. doi: 10.1016/j.clinph.2017.04.026.

15. Winterhalder M, Maiwald T, Voss HU, Aschenbrenner-Scheibe R, Timmer J, Schulze-Bonhage A. The seizure prediction characteristic: a general framework to assess and compare seizure prediction methods. Epilepsy Behav. 2003;4(3):31825. doi: 10.1016/S1525-5050(03)00105-7.

16. Wang L, Wang C, Fu F, Yu X, Guo H, Xu C, et al. Temporal lobe seizure prediction based on a complex Gaussian wavelet. Clin Neurophysiol. 2011;122(4):656-63. doi: 10.1016/j. clinph.2010.09.018.

17. Front Matter. In: Mallat S, ed. A Wavelet Tour of Signal Processing. 3rd ed. Boston: Academic Press; 2009. p. iii.

18. Sharabaty H, Martin J, Jammes B, Esteve D. Alpha and Theta Wave Localisation using Hilbert-Huang Transform: Empirical Study of the Accuracy. IEEE 2006 2nd International Conference on Information \& Communication Technologies; 2006. doi: 10.1109/ICTTA.2006.1684538.

19. Losonczi L, Bako L, Brassai ST, Márton LF. Hilbert-Huang transform used for EEG signal analysis. The 6th edition of the Interdisciplinarity in Engineering International Conference 2012; "Petru Maior" University of Tîrgu Mure区, Romania.

20. Huang NE, Shen Z, Long SR, Wu MC, Shih HH, Zheng Q, et al. The empirical mode decomposition and the Hilbert spectrum for nonlinear and non-stationary time series analysis. Proc R Soc Lond A. 1998;454(1971):903-95. doi: 10.1098/rspa.1998.0193.

21. Baghdadi G, Nasrabadi AM. EEG phase synchronization during 
hypnosis induction. J Med Eng Technol. 2012;36(4):222-9. doi: 10.3109/03091902.2012.668262.

22. Rosenblum M, Pikovsky A, Kurths J, Schäfer C, Tass PA. Chapter 9 Phase synchronization: from theory to data analysis. In: Moss F, Gielen S, eds. Handbook of Biological Physics. Vo.4. North-Holland: Elsevier; 2001. p. 279-321. doi: 10.1016/S1383-8121(01)80012-9.

23. Daniel WW. Applied nonparametric statistics. 2nd ed. Boston: PWS-KENT; 1990.

24. Soleimani H, Lucas C, Araabi BN, Schwabe L. Adaptive prediction of epileptic seizures from intracranial recordings. Biomed Signal Process Control. 2012;7(5):456-64. doi: 10.1016/j.bspc.2011.11.007.

25. Gath I, Geva AB. Unsupervised optimal fuzzy clustering. IEEE Trans Pattern Anal Mach Intell. 1989;11(7):773-80. doi: 10.1109/34.192473.

26. Soleimani H, Lucas C, Araabi BN. Recursive Gath-Geva clustering as a basis for evolving neuro-fuzzy modeling. International Conference on Fuzzy Systems; 2010. doi: 10.1109/FUZZY.2010.5584088.

27. Li S, Zhou W, Yuan Q, Liu Y. Seizure prediction using spike rate of intracranial EEG. IEEE Trans Neural Syst Rehabil Eng.
2013;21(6):880-6. doi: 10.1109/tnsre.2013.2282153.

28. Jang JS, Sun CT, Mizutani E. Neuro-Fuzzy and Soft Computing: A Computational Approach to Learning and Machine Intelligence. 1st ed. Pearson; 1997.

29. Van Leekwijck W, Kerre EE. Defuzzification: criteria and classification. Fuzzy Sets Syst. 1999;108(2):159-78. doi: 10.1016/S0165-0114(97)00337-0.

30. Arthurs S, Zaveri HP, Frei MG, Osorio I. Patient and caregiver perspectives on seizure prediction. Epilepsy Behav. 2010;19(3):474-7. doi: 10.1016/j.yebeh.2010.08.010.

31. Gadhoumi K, Lina JM, Gotman J. Seizure prediction in patients with mesial temporal lobe epilepsy using EEG measures of state similarity. Clin Neurophysiol. 2013;124(9):1745-54. doi: 10.1016/j.clinph.2013.04.006.

32. Zhang Y, Zhou W, Yuan Q, Wu Q. A low computation cost method for seizure prediction. Epilepsy Res. 2014;108(8):135766. doi: 10.1016/j.eplepsyres.2014.06.007.

33. Hung $\mathrm{SH}$, Chao CF, Wang SK, Lin BS, Lin CT. VLSI implementation for epileptic seizure prediction system based on wavelet and chaos theory. TENCON 2010 2010 IEEE Region 10 Conference; 2010. doi: 10.1109/ TENCON.2010.5686655. 